\title{
Towards The Utilization of Normalized LMS Algorithm in Adaptive Filter
}

\author{
Misbah Abdelsalam Misbah Emhammed \& Y. H Ho \\ Department of electronics \& computer engineering - University Technical Malaysia Melaka 76100 Hang Tuah \\ Jaya, Durian Tungga, Melaka, Malaysia
}

\begin{abstract}
The latest trends in reducing the environmental-noise related issues have received extra attention recently. The empirical studies reasoned this to the rapid growth in using technology in everyday things which in turn led to continues use of machines supported by noisy engines. Most of these machines results a high speed wind buffeting in which it effect the environment and people. Therefore, studies on reducing engine noise have become the main era where an alternative suggestions is still needed. In this paper, we focused on how the development of algorithms helped reduce the level of noise. This in turn led us to utilize the Least Mean Square (LMS) and Normalized Least Mean Square (NLMS) algorithms in order to do so. MATLAB was used for simulating the adaptive filter with a noisy tone signal and white noise signal. The analysis was mainly concentrated on the adapted algorithms' performance. This study provides the insights necessary for considering other filtering mechanisms based on the normalized least mean square.
\end{abstract}

Index Terms: least mean square, normalized least mean square, adaptive filter. noisy tone signal.

\section{Introduction}

Nowadays, the mechanisms for promoting active noise cancellation based on the utilization of different classes of adaptive filter have described by researchers as an alternative way for controlling the level of noise by generating the least $\mathrm{m}$. According to [1], this can be measured by direct microphone in which it found to accurately detect the total references and error signals. Determine the level of noise from both external and internal sources can help understand the level of effect on the performance and also to distortion of the sound resulted from the internal source. Hence, a wave separation technique is usually used in order to distinguish the effect resulted from the internal and external sources of sound. Using microphone for picking the noise in both sources is found to be undesirable. Such observation is reported by different scholars that microphone has a significant effect in reducing the quality of the audio signal [1]. As such, the importance of adopting an alternative mechanisms could result in effective removal of noise [2]. These in turn led researchers to suggest the use of additional adaptive filters to reduce the signal corruption resulted from the audio signal in terms of predictable and unpredictable noise. Adaptive filter provide a self-adjustment based on the process of transferring responses based on the level of optimization algorithm driven by an error signal. This led us to conclude that using adaptive filter make it possible to control the behavior in certain period of time. It also make it possible for the filter to be accustomed to the changes in function of the signal [3].

Based on the mentioned, the adaptive filter has been used by many researchers for the aim of maintaining a balanced performance and flexibility; this can be found in the applications of medical where it used to reduce information. Such process depends mainly on the way adaptive filter act when the input both from different signal sources would lead to control the actual frequency of the noise as it fluctuates. The reason is mainly back to that adaptive technique accepts sequences resulted with a smaller rejection range [4]. Moreover, the adaptive filter adjusts the noise level by removing the total noise from signal as shown in Fig 1A [5].

The separated adaptive filter help processing the reference signal $\mathrm{x}(\mathrm{n})$ as a variable filter in which noise cancelation algorithm is begin processed to generate $y(n)$. This process is carried by refereeing to the density of the filter's weights $w(n)$ as shown in Figure 1-B. However, the resulted $y(n)$ is deducted from the filter's weights by $d(n)$ in order to gain the error $e(n)$. The primary sensor receives noise $x 1(n)$ which has correlation with noise $\mathrm{x}(\mathrm{n})$ in an unknown way. In this study, we aim to concentrate on the mechanism for reducing the error signal $\mathrm{e}(\mathrm{n})$. 


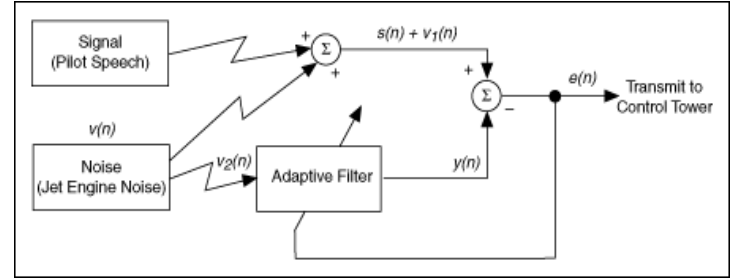

Fig 1-A. Adaptive noise canceller

The process presented in Figure 1-B serves as a foundation for particular adaptive filter realizations, such as LMS; NLS; and Recursive Least Square (RLS) algorithms [6]. The idea behind the block diagram is that a variable filter extracts an estimate of the desired signal. Researchers suggested that adaptive algorithms such as LMS is the most effective way for controlling noise reduction in which it enable good tracking capabilities and simplicity in stationary environment.

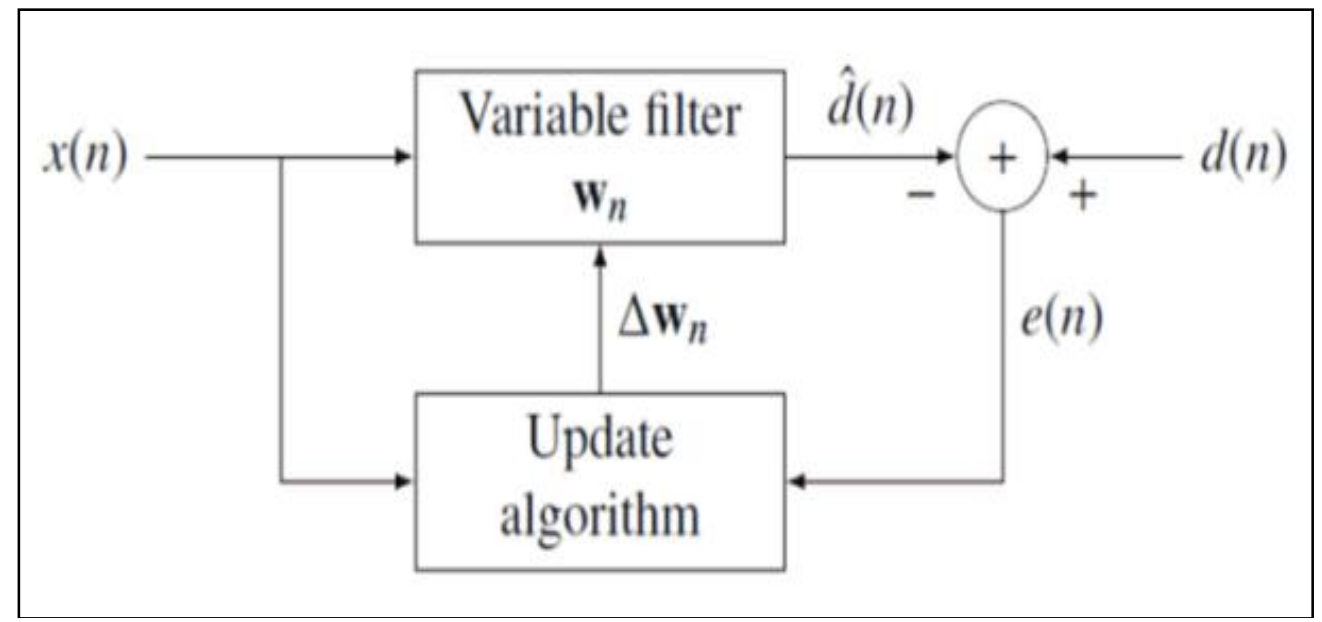

Figure 1-B: Adaptive filter realizations

\section{A. Steepest Descent Algorithm}

\section{Design Of Adaptive Filter}

This study aimed at determining the effect of utilizing an adaptive filter to resolve problems associated with the tap-weight vector with different distribution flows. In order to do so, a steepest descent algorithm is used as an optimization algorithm to determine the local minimum of a function where the separated function takes steps proportional to the negative source. This process is obtained as follows:

The total weight of initial vector is identified as a 0 , which leads to $\mathrm{w}(0)=$ null vector.

Based on this, we calculated the total gradient vector, which we measured as the total mean of gradient in the squared error of $J(n) w r t w(n)$ at time $n$.

Then the researcher determined the next estimation at the tap-weight vector by adjusting the first obtained estimation in different direction to the one of the gradient vector.

In order to loop the vector, we relied on this question where:

$\mathrm{w}(\mathrm{n}+1)=\mathrm{w}(\mathrm{n})+0.5 \mu[-\nabla(\mathrm{n})]$,

$\mu=$ positive real-valued constant .....(1)

$\nabla(\mathrm{n})=-2 \mathrm{p}+2 \mathrm{Rw}(\mathrm{n}) \ldots .(2)$

Meanwhile, in order to estimate the total vector error generated from the signal, we relied on the steepest-descent algorithm where the value of correlation matrix $\mathrm{R}$ and cross-correlation matrix $\mathrm{p}$ are identified. This was carried by retrieving the values resulted from filter signals named which known as a reference and primary processes as shown in the following equation:

$\mathrm{w}(\mathrm{n}+1)=\mathrm{w}(\mathrm{n})+\mu[\mathrm{p}-\mathrm{Rw}(\mathrm{n})] \mathrm{n}=0,1,$.

The resulted weight led us to conclude that parameter $\mu$ help adjusts the total size of the incremental correction found in the tap-weight vector. Hence, $\mu$ is known as the parameter that controls the step-size or weight generated from the previous process [7]. 


\section{B. Least-Mean-Squares (LMS) Algorithm}

As mentioned earlier, adaptive filter is consider as a self-adjust based on an adaptive algorithm. The LMS algorithm is known as an adaptive algorithm which works at reducing the coefficients of filters where the total power resulted is minimized from the total error resulted from the original signal. Therefore, processing a fast convergence leads always to short time that adaptive filter take to determine the coefficients of the filter suitable for adjusting the power of the error signal. In addition, the time required for processing the error signal is measured based on the period that adaptive filters take to converge [8].

In this study, we relied on the estimation error to predicate the weights of the adjusted filter. Based on the literature, we found that step-size parameter $\nabla$ is appropriate for measuring the gradient vector at each iteration, where the tap-weight vector is also processed in the following operation based on the steepest-descent method. The use of this method was to descend along the error signal by captivating a new direction of the gradient of that function. This led to the following equation:

$$
\text { Gradient vector, } \nabla(\mathrm{n})=-2 \mathrm{p}+2 \mathrm{Rw}(\mathrm{n})
$$

To estimate this, we calculated the total correlation matrix $\mathrm{R}$ and cross-correlation matrix $\mathrm{p}$ by immediate generalization of the vector value where:

$$
\begin{gathered}
\mathrm{R}^{\prime}(\mathrm{n})=\mathrm{u}(\mathrm{n}) \mathrm{u}(\mathrm{n}) \ldots(4) \\
\mathrm{p}^{\prime}(\mathrm{n})=\mathrm{u}(\mathrm{n}) \mathrm{d}^{*}(\mathrm{n}) \ldots(5)
\end{gathered}
$$

This in turn led us to suggest the instantaneous estimate of the gradient-vector based on the following equation:

$\nabla(n)=-2 u(n) d^{*}(n)+2 u(n) u H(n) w(n) \ldots(6)$

Changing the resulted value from the previous estimation with considering the steepest-descent algorithm help us to determine the recursive relation for adjusting the value of tap-weight vector based on the following equation:

$w^{\prime}(n+1)=w^{\prime}(n)+\mu u(n)\left[d^{*}(n)-u H(n) w^{\prime}(n)\right]$

Furthermore, the modified LMS's equation for this study was carried in the form of a pair of relations measured by the following equations:

$\mathrm{e}(\mathrm{n})=\mathrm{d}(\mathrm{n})-\mathrm{uH}(\mathrm{n}) \mathrm{w}^{\prime}(\mathrm{n})$

$\mathrm{w}^{\prime}(\mathrm{n}+1)=\mathrm{w}^{\prime}(\mathrm{n})+\nabla \mathrm{u}(\mathrm{n}) \mathrm{e}^{*}(\mathrm{n})$

Finally, we also considered the total mean value of overload within squared error by the actual value of $\mathrm{J}(\infty)$ which found to be more than Jmin, shorted condition .The shorted condition of $\mathrm{M}$ is measured in this study as the dimensionless ratio that covers the initial value of the steady-state carried by excess mean-squared error which represented by the following equation:

$\mathrm{M}=\mu \sum \frac{\gamma \mathrm{i}}{2}-\mu \sum \gamma \mathrm{i} \ldots .(10)$

Based on all the mentioned equations, we tested the ratio based LMS in MATLAB where the result is generated and discussed in the next section. 


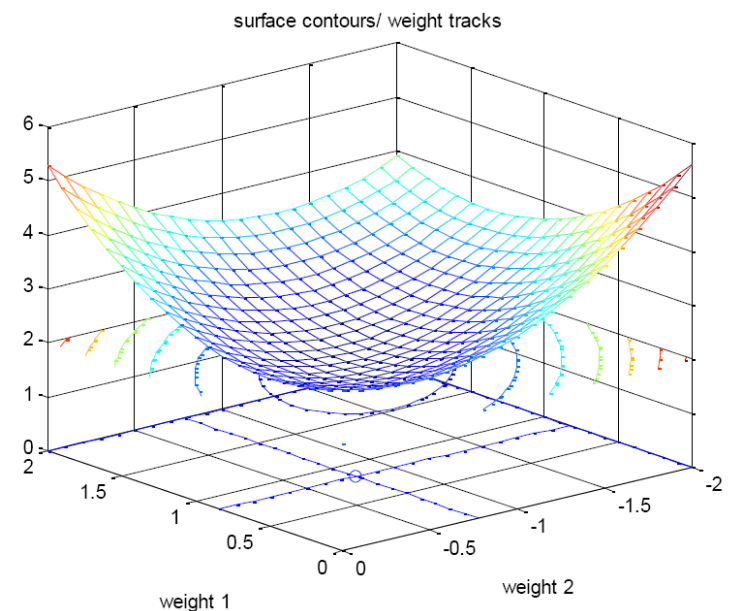

Fig 2. Surface shape/ weight tracks

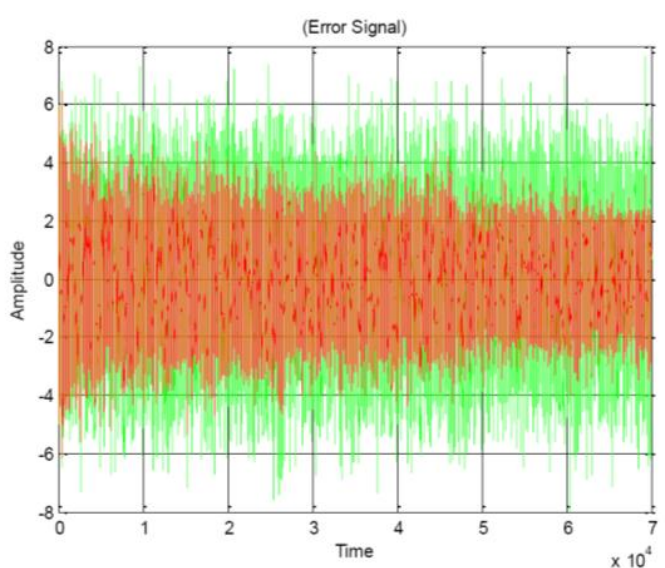

Fig 3 . Total error signal
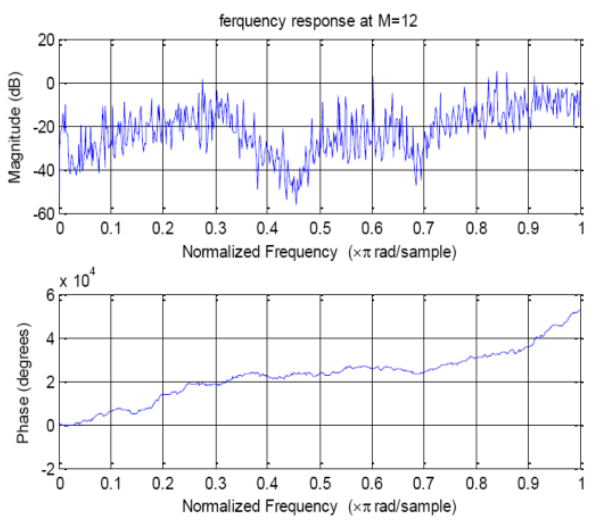

Fig 4. Total incidence response 1

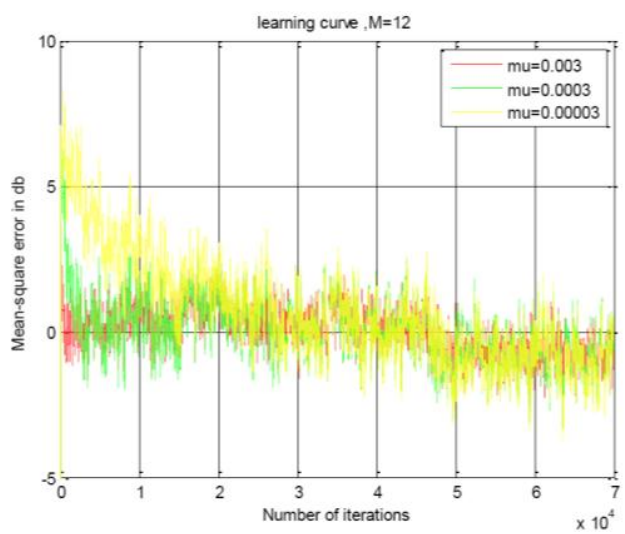

Fig 5. Learning based mean of error square curve 1
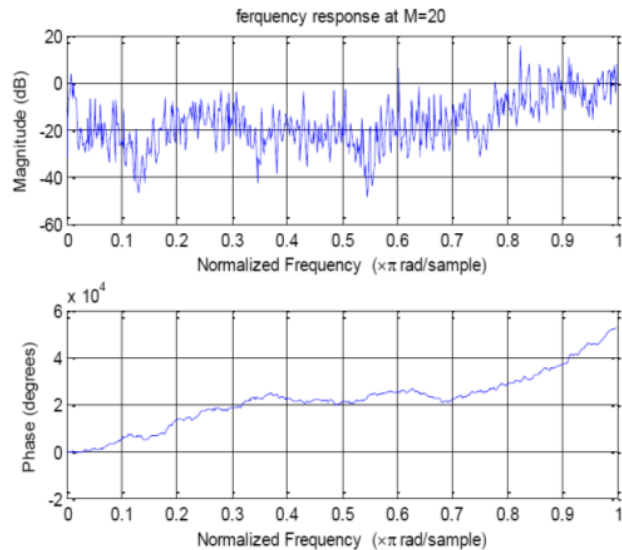

Fig 6. Incidence response 2 


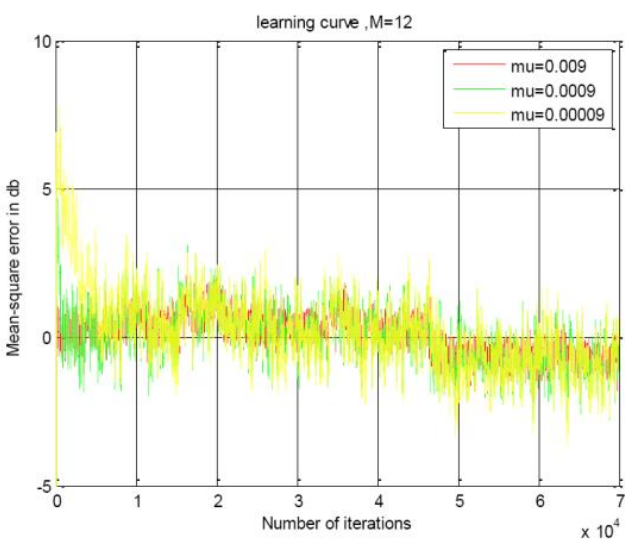

Fig 7. Learning based mean of error square curve 2

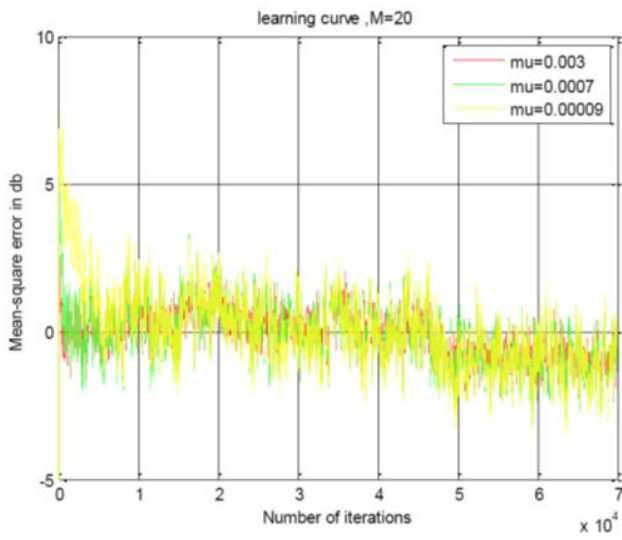

Fig 8. Learning curve 3

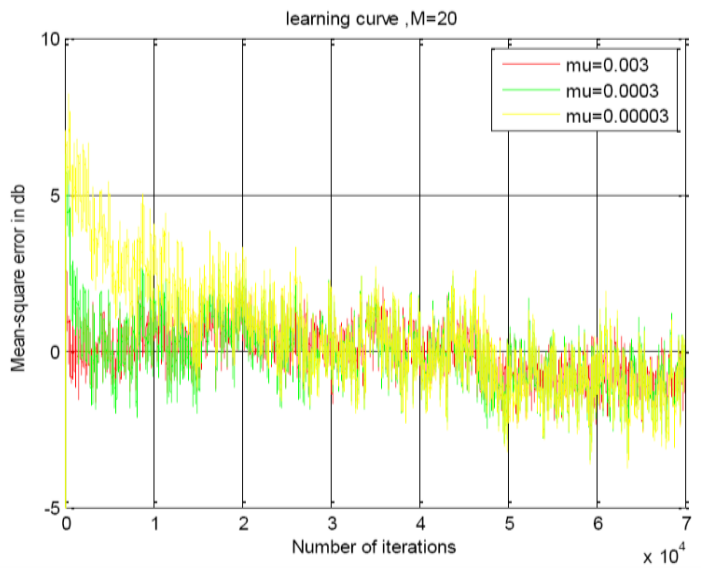

Fig 9. Learning based mean of error square curve 4

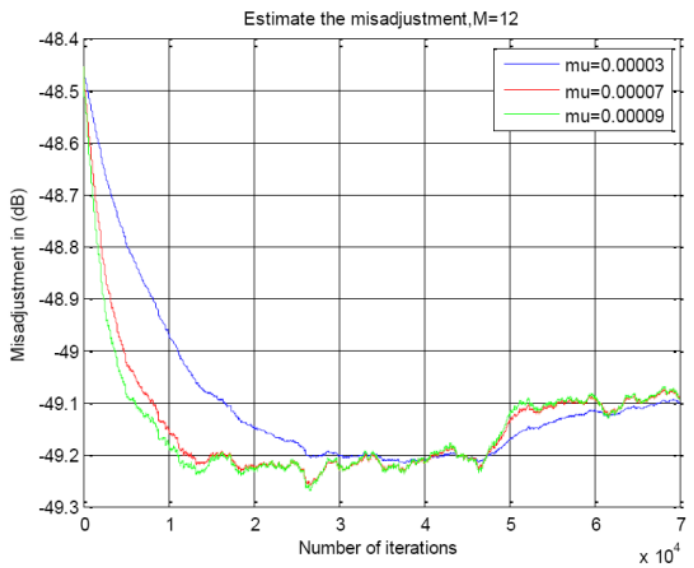

Fig10. Shorted condition curve 1

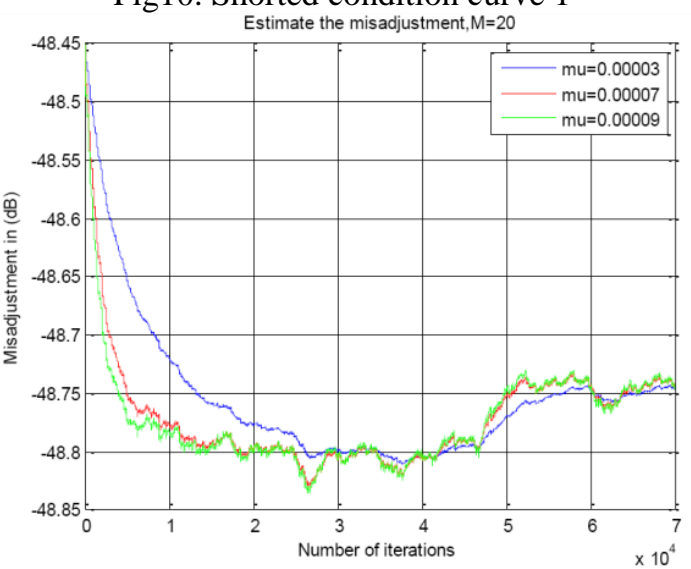

Fig 11. Shorted condition curve 2

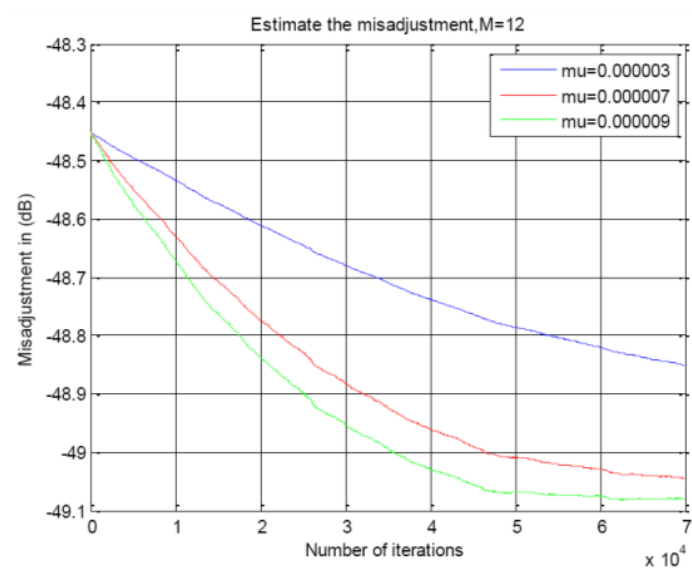

Fig 12. Shorted condition curve 3

\section{Result And Discussion}

The result obtained in the previous section shows the performance of the adaptive filtering. In this study, we measured the surface shape, incidence response, learning based mean square error curves and shorted condition curves for the three scenarios. We relied on the mean square error for measuring the LMS performance associated with the total number of vectors in the adaptive filter. In addition, we adapted a mechanism by [2] to measure the filter step size of $\mu$ based on the iterations resulted from performing the function MSE. The rationality for using MSE back to that this function simplify the process of decreasing iteration within vectors, this also consider the total weight and square error that result from the learning of different values of step size.

Therefore, a simulation based measure was adapted to determine the ability of the LMS algorithm in reducing the resulted noise from the audio signals. The performance analysis revealed that iterations for noisy 
tonal signal to mean square error for various step-size parameters. The result showed that adaptive filter with large step-size of $\mu$ result unbalanced activities which found to be associated with the error output and error signals. However, we also found that $\mu$ of 0.0003 in the second scenario was not yet converged after a number of iterations. Therefore, in the case of 0.0003 the balance between iterations remains stable at 1000 samples as shown in Table 1. Based on the comparison result, we found that simulating adaptive filter for noise reduction is controlled by the range of $\mu$ values. Finally, this result showed how LMS contributes to the stability of filter based on the total percept of noise reduction in MSE.

Table 1: Performance comparison of LMS and NLMS

\begin{tabular}{ccccc}
\hline S.N & algorithm & MSE & $\begin{array}{c}\text { \%noise } \\
\text { reduction }\end{array}$ & stability \\
\hline 1 & LMS & $2.5 \mathrm{e}-002$ & $91.6 \%$ & Highly stable \\
2 & NLMS & $2.1 \mathrm{e}-002$ & $93.8 \%$ & high \\
\hline
\end{tabular}

\section{Conclusion}

This study carried the process for utilizing adaptive filter based on the LME and NLMS algorithms for the aim of stable reduction of noise in audio signals. This study also showed the main procedures for measuring the noise reduction based on the utilization of ten equations discussed realer. MATLAB was used after to determine the performance of LMS algorithm. The result revealed that using LMS help increasing noise cancelation. The noise attached with the audio signal was measured and compared in Table 1 . The comparison result provides the necessary insights about the effects of utilizing LMS and NLMS in operating the adaptive filter.

\section{Acknowledgment}

My sincere thanks go to PROFESOR DATUK DR. Ahmad Yusoff bin Hassan and PROFESOR DR. Mohd Razali bin Muhamad for their support.

\section{References}

[1]. D. B. Chonde, N. Abolmaali, G. Arabasz, A. R. Guimaraes, and C. Catana, "Effect of MRI acoustic noise on cerebral fludeoxyglucose uptake in simultaneous MR-PET imaging," Investigative radiology, vol. 48, pp. 302-312, 2013.

[2]. C. Quince, A. Lanzen, R. J. Davenport, and P. J. Turnbaugh, "Removing noise from pyrosequenced amplicons," BMC bioinformatics, vol. 12, p. 38, 2011

[3]. P. Dean, J. Porrill, C.-F. Ekerot, and H. Jörntell, "The cerebellar microcircuit as an adaptive filter: experimental and computational evidence," Nature Reviews Neuroscience, vol. 11, pp. 30-43, 2010.

[4]. R. K. Thenua and S. Agarwal, "Simulation and Performance Analyasis of Adaptive Filter in Noise Cancellation," International Journal of Engineering Science and Technology, vol. 2, pp. 4373-4378, 2010.

[5]. S. Griffin, A. Weston, and J. Anderson, "Adaptive noise cancellation system for low frequency transmission of sound in open fan aircraft," Shock and Vibration, vol. 20, pp. 989-1000, 2013.

[6]. K. Wagner and M. Doroslovacki, "Proportionate-type normalized least mean square algorithms with gain allocation motivated by mean-square-error minimization for white input," Signal Processing, IEEE Transactions on, vol. 59, pp. 2410-2415, 2011.

[7]. Y. Guo and J. Ji, "Variable Momentum Factor Decision Feedback Blind Equalization Algorithm Based on Constant Parameter Error Function," in Information and Automation, ed: Springer, 2011, pp. 282-288.

[8]. K. H. Ahmed, A. M. Massoud, S. J. Finney, and B. W. Williams, "A modified stationary reference frame-based predictive current control with zero steady-state error for LCL coupled inverter-based distributed generation systems," Industrial Electronics, IEEE Transactions on, vol. 58, pp. 1359-1370, 2011. 